\title{
Filogeografía del complejo Caesalpinia hintonii: (Leguminosae: Caesalpinioideae: Poincianella)
}

\section{Phylogeography of the Caesalpinia hintonii complex (Leguminosae: Caesalpinioideae: Poincianella)}

\author{
Solange Sotuyo ${ }^{1 *}$, Alfonso Delgado-Salinas ${ }^{1}$, Gwilym P. Lewis ${ }^{2}$, Mark W. Chase ${ }^{3}$, Luca Ferrari ${ }^{4}$ y Ken \\ Oyama $^{5}$
}

${ }^{1}$ Departamento de Botánica, Instituto de Biología, Universidad Nacional Autónoma de México. Ciudad Universitaria 04510 México, D. F., México.

${ }^{2}$ The Herbarium, Royal Botanic Gardens. Kew, Richmond, Surrey, TW9, 3AB, UK.

${ }^{3}$ Jodrell Laboratory, Royal Botanic Gardens. Kew, Richmond, Surrey, TW9, 3SD, UK.

${ }^{4}$ Centro de Geociencias, Universidad Nacional Autónoma de México, Campus Juriquilla 76230 Querétaro, Querétaro, México.

${ }^{5}$ Centro de Investigaciones en Ecosistemas, Universidad Nacional Autónoma de México. Antigua Carretera a Pátzcuaro 8701, Col. Ex Hacienda de

San José de la Huerta, 58190 Morelia, Michoacán, México.

*Correspondencia: jssotuyo@ibunam2.ibiologia.unam.mx

Resumen. A pesar del crecido número de estudios que se ha realizado para explorar la estructura genética y filogeográfica en especies mexicanas, hay carencia de estudios para especies de selva baja, zonas con alto grado de endemismo. Para un mejor entendimiento de dichas áreas se realizó un estudio filogeográfico y el fechamiento de las especies del complejo Caesalpinia hintonii distribuido en la depresión del río Balsas y el valle de Tehuacán-Cuicatlán. Se determinó la estructura filogeográfica del complejo analizando las secuencias de 4 regiones de plastidio (intrón $\operatorname{trn} L$, espaciador trnL-F, espaciador $p s b A$-trnH y espaciador $a c c D-p s a I$ ) en todas las poblaciones disponibles. Se estimó la fecha del origen del complejo mediante un método no paramétrico (NPRS). Las diversidades haplotídicas (0.64-1) y las diferencias nucleotídicas (3.811.52) en todas las especies fueron altas respecto a valores registrados para otras plantas. Las poblaciones de C. oyamae, morfológicamente indistintas están genéticamente estructuradas y presentan 3 haplogrupos donde el flujo génico entre ellos es bajo y se consideraron entidades crípticas. Las poblaciones de C. hintonii son un grupo genéticamente homogéneo a pesar de ser morfológicamente distintas. Las poblaciones de C. macvaughii forman 2 linajes divergentes entre los que existe flujo génico $(\mathrm{Nm}=1.41)$. Los eventos de divergencia en 2 grandes linajes al este y oeste del río Balsas pueden explicarse por eventos geológicos. Los resultados indican que el complejo se encuentra bajo divergencia y en algunos casos radiación morfostática.

Palabras clave: depresión del río Balsas, fechamiento, $\operatorname{trn} L$, $\operatorname{trn} L-F$, $p s b A-\operatorname{trnH}$, accD-psaI, México, valle de TehuacánCuicatlán.

\begin{abstract}
Although many phylogeographic studies have been conducted to analyze the genetic and phylogeographic structure of Mexican species, such studies are nearly absent for plants of dry seasonal forest, precincts with high level of endemism. To better understand this areas, we undertook a phylogeographic study and dating were carried out on the Caesalpinia hintonii complex distributed in the Río Balsas Depression and Tehuacán-Cuicatlán Valley. The phylogeographic structure was determined analyzing sequences from 4 plastid regions ( $\operatorname{trn} L$, $\operatorname{trn} L-F$, $p s b A$-trnH and $a c c D$ psal) in all populations. A nonparametric rate smoothing (NPRS) method was employed to estimate the time of origin of the species complex. Haplotypic (0.64-1) and the nucleotide differences (3.8-11.52) were high in all species. The morphologically indistinct $C$. oyamae populations are genetically structured and showed 3 haplogroups where gene flow is low; considered them here as cryptic entities. The $C$. hintonii populations are genetically close-knit, even though they are morphologically distinct. The populations of $C$. macvaughii exhibit 2 divergent lineages with gene flow $(\mathrm{Nm}=1.41)$. Divergence events in 2 lineages between east and west from Rio Balsas Depression can be explained by geologic events. Our results showed that the $C$. hintonii complex is under diversification and in some cases morphostatic radiation.
\end{abstract}

Key words: Río Balsas Depression, cryptic species, dating, trnL, trnL-F, psbA-trnH, accD-psaI, Mexico, TehuacánCuicatlán Valley.

Recibido: 29 septiembre 2009; aceptado: 17 marzo 2010 


\section{Introducción}

El crecido número de estudios filogeográficos se han desarrollado en México en los últimos años (e.g., Matos y Schaal, 2000; Nason et al., 2002; González-Rodríguez et al., 2004; Tovar-Sánchez y Oyama, 2004) se han enfocado en especies de regiones montañosas y desérticas pero no en las de zonas de selvas secas. Por otro lado, la necesidad de estudiar las selvas secas contando con el conocimiento filogenético de sus especies como parte esencial para entender su estructura y endemismo (Lavin, 2006), y la sugerencia hecha por Pennington (2004) de que la distribución geográfica actual de las selvas secas en el neotrópico debe ser considerada en relación con las fluctuaciones climáticas del Cuaternario dan la pauta para plantear estudios que traten de entender la estructura y evolución de este tipo de vegetación.

En México es conocido que la depresión del río Balsas (DRB) y el valle de Tehuacán-Cuicatlán (VTC) contienen una amplia zona de selva baja y un gran número de taxa endémicos (Sousa y Delgado, 1993; Becerra y Venable, 1999). La naturaleza de ese endemismo y el papel que desempeñan los cambios climáticos en la tasa de evolución y especiación en la zona aún no han sido explorados. Lo anterior probablemente sea por la complejidad de patrones esperados en las selvas secas y a que los ciclos de contracción y expansión debieron ser limitados en comparación con las áreas del norte.

La DRB y VTC se localizan en la parte centro occidental del país, forman parte de la planicie morfotectónica de la sierra Madre del Sur e incluyen los estados de Guerrero, Morelos, Puebla y Oaxaca.

La DRB está delimitada al oriente por el escudo mixteco que une la sierra Madre del Sur con el Eje Neovolcánico o Faja Volcánica Transmexicana (FVTM); al norte con el Eje Neovolcánico desde los límites de Michoacán y Jalisco hasta el volcán de la Malinche (Puebla-Tlaxcala); al este con la sierra Madre de Oaxaca; al sur y al oeste con la sierra Madre del Sur. La DRB se dividide en la depresión oriental y la depresión occidental (Toledo, 1982) por un estrechamiento (lengüeta de esquistos del complejo basal) ocasionado por la sierra de Taxco-Teloloapan (Guerrero, Cabral-Cano et al., 2000).

La DRB es un gran sinclornio que comenzó a levantarse en el Mesozoico y posteriormente se plegó para formar la depresión (Terciario). La depresión se formó como una meseta de rellenamiento cerrado, debido a los movimientos tectónicos y por la presencia de numerosas fallas. La meseta contenía una serie de lagos internos, pero los movimientos orogénicos que afectaron la sierra Madre del Sur originaron fracturas en la cuenca, convirtiéndose en una meseta de denudación.
La menor precipitación ocurre en las zonas bajas (cercanas al río), que además de ser las más cálidas, son también las más secas. La variación altitudinal de la cresta de la sierra Madre del Sur influye significativamente en la distribución longitudinal de la humedad (Toledo, 1982) por lo que la parte más seca de la depresión se encuentra cerca de la desembocadura del río, donde la sierra tiene un mínimo de altitud; la parte más humeda, a su vez, se localiza a los $100^{\circ}$, que es el filo más alto de la sierra de Taxco-Teloloapan. La depresión oriental (Puebla Oaxaca) presenta una tercera zona de poca humedad que está separada del cañón del Zopilote por una franja más húmeda (Jiménez, 1980).

Por otra parte el VTC se localiza entre los estados de Puebla y Oaxaca. Las fases de formación del VTC son producto de la falla de Oaxaca durante el levantamiento progresivo de la sierra Mazateca y el hundimiento relativo del valle de Tehuacán ocurrido desde el Eoceno hasta el Cuaternario. La historia geológica comprende 4 fases: 1) la formación del complejo basal que data del PaleozoicoMesozoico; 2) una inmersión de la cuenca a principios del Cretácico inferior, la cual formó el portal del Balsas y el aislamiento del valle de Tehuacán respecto al golfo de México con una depositación de sedimentos marinos ricos en material salino; 3) una serie de movimientos orogénicos que aíslan la cuenca y por tanto, el VTC pierde conexión con la DRB dando origen a un lago endorréico que data del Cretácico inferior, y 4) la captación por parte del río Santo Domingo, afluente del río Papaloapan, del río Salado y sus tributarios, por erosión resonante (Beristain et al., 1996). Estos procesos formaron cerros de cimas planas con rocas calizas como roca madre, zonas de ladera con lutitas, zonas de depositación aluvial a lo largo del río Salado, así como zonas de material ígneo (Ferrusquía-Villafranca, 1996).

Para examinar los procesos de especiación en la DRB y VTC podríamos valernos de 2 hipótesis: la de vicarianza y la de dispersión. Bajo la hipótesis de vicarianza, la existencia de distintos linajes alopátricos en la DBR y VTC podría estar reflejando la desaparición de la barrera que fragmentó la población ancestral, pero las "especies" reunidas podrían considerarse hermanas y su origen históricamente ligado. Es decir, bajo este escenario de especiación alopátrica, los cambios climáticos explicarían la disyunción de las áreas de distribución y la subsiguiente evolución en linajes separados. Sin embargo, bajo la hipótesis de dispersión, los taxa alopátricos de la DBR y VTC no necesariamente se encontrarían ligados temporalmente. La dispersión de los linajes durante las glaciaciones del Pleistoceno podrían haber homogeneizado los haplotipos de estos taxa haciendo que en la actualidad muestren baja diversidad genética y divergencia comparados con los taxa que no estuvieron expuestos a los desplazamientos y 
homogeneizaciones del Pleistoceno.

En el presente trabajo, se eligió el complejo Caesalpinia hintonii como modelo de estudio para determinar los patrones filogeográficos de la región de la DRB y del VTC. Se reconocen 6 especies endémicas dentro del complejo Caesalpinia hintonii, distribuidas disyuntamente en la DRB y VTC (Sotuyo y Lewis, 2007). El complejo C. hintonii se considera un grupo monofilético cuya especie hermana es C. pannosa que se distribuye en Baja California Sur (Sotuyo et al. 2007). La densidad poblacional de todas las especies es baja y se distribuyen a manera de manchones a lo largo de la DRB y el VTC. La morfología floral de todas ellas es similar lo que ha originado problemas para circunscribirlas (Sotuyo et al., 2004). De acuerdo con J. L. Contreras (com. pers.), las abejas que visitan sus flores pertenecen a las mismas especies, Centris (Xerocentris) nitida, Xylocopa mexicanorum y un taxon no identificado (R. Ayala, com. pers.), lo cual podría suponer la probabilidad de flujo génico entre ellas.

Dada la historia geológica de la región y la gran biodiversidad que alberga, donde se reconocen altos niveles de endemismo, entre ellos los de la familia Leguminosae, se consideró que el estudio de los patrones de divergencia de grupos de especies, como el complejo $C$. hintonii, podría ser significativo para el entendimiento de los procesos de especiación que han tenido lugar en ella.

Los objetivos de este estudio fueron: 1) la determinación de la estructura filogeográfica de las poblaciones de todas las especies del complejo Caesalpinia hintonii localizadas en la DRB y en el VTC, y 2) las estimaciones del tiempo de divergencia de C. pannosa, especie hermana del complejo $C$. hintonii, y de la edad del complejo para saber qué tan reciente fue su diversificación. Además, se calculó la edad de los linajes encontrados por Sotuyo (2007) en las porciones oriental y occidental de la DRB. Se trató de asociar todas las edades obtenidas con algún evento geológico ocurrido dentro de la DRB y en el VTC, formando parte de la planicie morfotectónica de la sierra Madre del Sur (Ferrusquía-Villafranca 1993).

\section{Materiales y métodos}

El complejo Caesalpinia hintonii comprende 6 especies: C. epifanioi J. L. Contreras, C. hintonii Sandwith, C. laxa Benth., C. macvaughii J. L. Contreras et G. P. Lewis, C. melanadenia (Rose) Standley y C. oyamae Sotuyo et Lewis, pertenecientes al denominado grupo taxonómico Poincianella (Sotuyo y Lewis, 2007). Además, C. pannosa se considera , especie hermana (Sotuyo et al. 2007) (Fig. 1). Para 43 individuos de 13 poblaciones se amplificaron el intrón trnL (Taberlet et al., 1991) y los espaciadores intergénicos trnL-F (Taberlet et al., 1991), psbA-trnH (Hamilton, 1999) y accD-psaI (Mendenhall, 1994) siguiendo los protocolos citados en Sotuyo et al. (2007). Todas las secuencias fueron depositadas en el GenBank® bajo los números DQ208712-DQ208930. Debido a que las regiones de plastidio usadas se heredan sin recombinación y comparten una misma historia, los datos de las 4 regiones analizadas, a menos de que se indique lo contrario, fueron combinados.

Variación y estructura genética. Para el análisis de variación se estimaron índices de diversidad nucleotídica y haplotípica con el programa DnaSP (DNA Sequence Polymorphism, versión 3.99; Rozas et al., 2003). El polimorfismo nucleotídico fue estimado como: S (sitios variables únicos), $\mathrm{K}$ (número promedio de diferencias nucleotídicas pareadas), $\pi$ (diversidad nucleotídica, Nei, 1987), h (número de haplotipos) y Hd (diversidad haplotídica). La diferenciación genética entre poblaciones se estimó mediante el número promedio de diferencias nucleotídicas por sitios entre las poblaciones (Dxy, Nei, 1987). También se calcularon los estadísticos Hs y Ks (Hudson et al., 1992), así como, Kxy y el número neto de substituciones nucleotídicas por sitio entre poblaciones (Da), (Nei, 1987). Se realizó un análisis de permutación (Hudson etal., 1992) para determinarcuándo se encontraban significativamente diferenciadas las poblaciones.

Las estimaciones de flujo génico se realizaron mediante el $\mathrm{F}_{\mathrm{ST}}$ (Hudson et al., 1992) para evaluar el número de migrantes entre poblaciones $(\mathrm{Nm})$. Se empleó una aproximación jerárquica (AMOVA; Excoffier et al., 1992) para calcular la varianza molecular en el complejo C. hintonii utilizando el programa Arlequin ver. 2006 (Excoffier et al., 2005). Las regiones se analizaron por separado y concatenadas.

Redes de haplotipos. Se generaron las matrices de haplotipos utilizando el programa DnaSP (Rozas et al., 2003); las inserciones/deleciones o indels no fueron considerados para el análisis pues el incluirlas como un quinto estado no generó redes distintas, únicamente un mayor número de pasos mutacionales. Posteriormente se empleó el programa NETWORK ver. 4.2.0.0 (Polzin y Daneschmand, 2003, http://www.fluxusengineering. com) para obtener la red de haplotipos (median networks, Bandelt et al., 1999).

Calibración molecular. Caesalpinia no posee un rico registro fósil debido a su escasa preservación y la carencia de estructuras morfológicas diagnósticas para el grupo (Herendeen y Crane, 1992). Dado que con fósiles recientes como $C$. spokanensis $(\approx 16.517$ millones de años, Ma) no existe certeza de la posición del árbol donde deben establecerse los puntos de calibración internos; las calibraciones se hicieron con puntos externos al grupo 


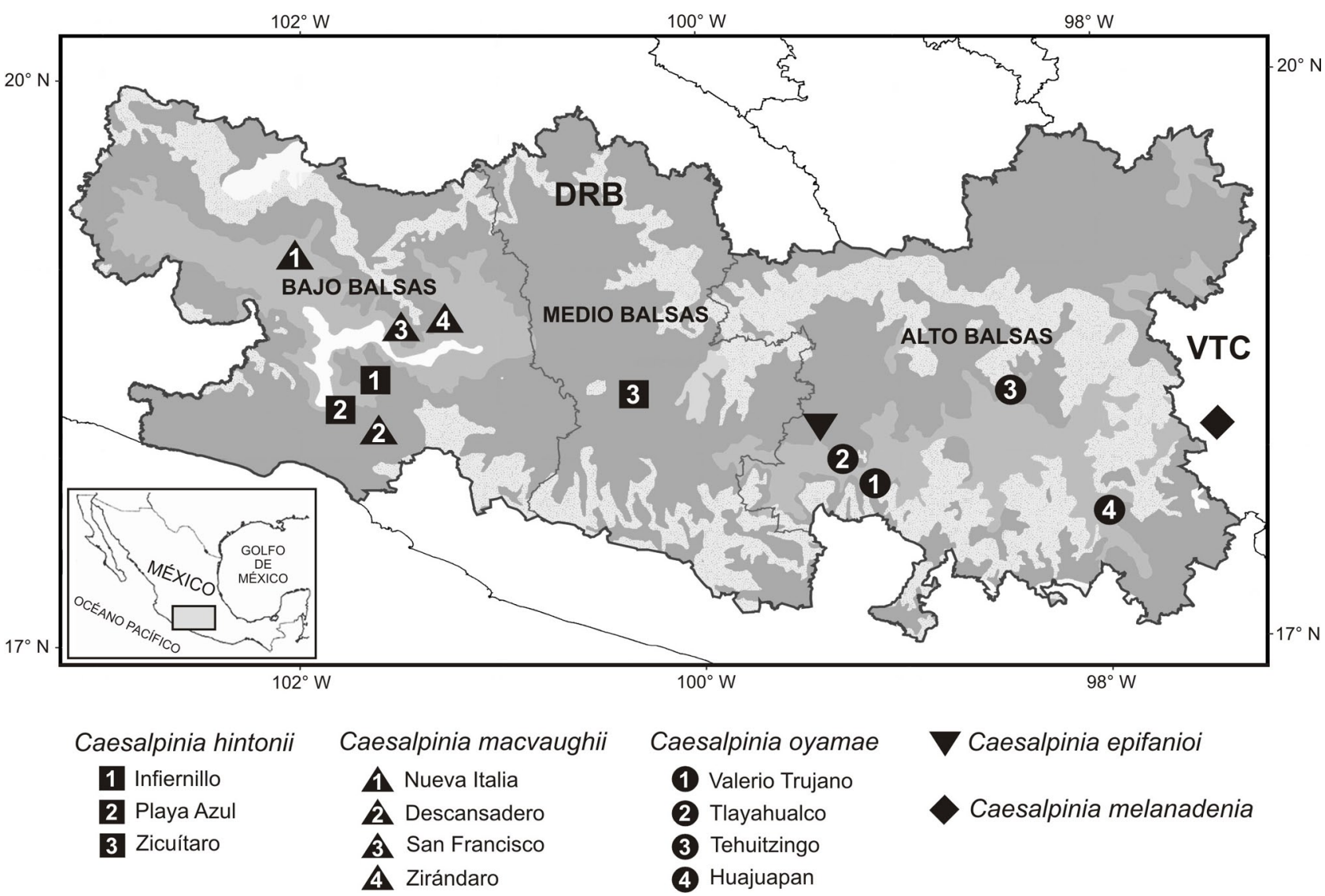

Figura 1. Localización de las poblaciones y especies del complejo Caesalpinia hintonii colectadas. DRB, depresión del río Balsas; VTC, valle de Tehuacán-Cuicatlán.

Poincianella, utilizando 2 fósiles, $C$. claibornensis y $C$. flumen-viridensis (Eoceno $\approx 45 \mathrm{Ma}$ ), pertenecientes al subgénero Mezoneuron (Herendeen, 1992; Herendeen y Crane, 1992).

Se empleó la matriz y los árboles generados para 175 taxa del género Caesalpinia, sensu lato, con secuencias del intrón trnL y el espaciador trnL-F (Sotuyo, 2007). Primero, se estimó la tasa de constancia entre linajes en el grupo de datos, en donde la hipótesis nula de constancia de la tasa evolutiva fue rechazada $(\mathrm{P}<0.05)$ aplicando el likelihood ratio test statistic $(\delta=2 \log \Lambda)$. El modelo de substitución que mejor se ajustó al grupo de datos se determinó utilizando el programa Modeltest ver. 3.06 (Posada y Crandall, 1998) en conjunción con el análisis filogenético usando parsimonia (PAUP, Swofford, 1998), estimando las topologías y la longitud óptima de ramas mediante el criterio de máxima verosimilitud (ML). Elegido el modelo de ML, se evaluó empleando el LR test (Felsenstein, 1981). Para estimar los tiempos de divergencia se usó el método nonparametric rate smoothing (NPRS, Sanderson, 1997) utilizando el programa TreeEdit v er.1.0a4-61 (Rambaut y Charleston, 2000) para generar los árboles ultramétricos. Finalmente, para calcular el error estándar de las estimaciones moleculares, fueron generadas 100 matrices bootstrap utilizando el árbol que se empleó para inferir los tiempos de divergencia. A partir de los 100 árboles ultramétricos generados con dichas matrices se calculó la desviación estándar a partir de las edades absolutas, contando con el valor SDV $=5.2725$.

\section{Resultados}

Diversidad nucleotídica (Cuadro 1). La diversidad haplotídica (Hd) dentro del complejo C. hintonii osciló entre 0.643 en la población Zicuítaro de $C$. hintonii, y 1.0 en las poblaciones de Valerio Trujano y Tehuitzingo de C. oyamae y Playa Azul de C. hintonii, así como en las poblaciones de C. melanadenia, C. epifanioi y C. pannosa. La diversidad nucleotídica $(\pi)$ dentro de las poblaciones 
Cuadro 1. Análisis de la diversidad genética para el complejo Caesalpinia hintonii. N, número de individuos; $P$, sitios variables (polimórficos); $S$, sitios variables únicos; $h$, número de haplotipos; $H d$, diversidad haplotípica; $\pi$, diversidad nucleotídica; $K$, promedio de diferencias nucleotídicas

\begin{tabular}{llllllll}
\hline Especie/Población & $N$ & $P$ & $S$ & $h$ & $H d$ & $\pi$ & $K$ \\
\hline C. oyamae & 25 & 58 & 25 & 20 & 0.980 & 0.006 & 10.520 \\
Valerio Trujano & 7 & 19 & 8 & 7 & 1.0 & 0.003 & 7.524 \\
Tehuitzingo & 5 & 10 & 8 & 5 & 1.0 & 0.002 & 4.400 \\
Tlayahualco & 9 & 10 & 8 & 7 & 0.917 & 0.001 & 2.667 \\
Huajuapan & 4 & 19 & 12 & 3 & 0.833 & 0.006 & 10.667 \\
C. hintonii & 17 & 17 & 15 & 9 & 0.787 & 0.002 & 2.206 \\
Infiernillo & 7 & 24 & 23 & 6 & 0.952 & 0.004 & 7.048 \\
Zicuítaro & 8 & 15 & 15 & 4 & 0.643 & 0.002 & 3.750 \\
Playa Azul & 2 & 9 & 9 & 2 & 1.0 & 0.010 & 9.0 \\
C. macvaughii & 10 & 0 & 0 & 1 & 0.905 & 0.006 & 11.524 \\
Descansadero & 3 & 25 & 25 & 2 & 0.667 & 0.009 & 16.667 \\
La Huacana/San Francisco & 3 & 0 & 0 & 1 & - & - & - \\
Zirándaro & 4 & 37 & 36 & 3 & 0.833 & 0.010 & 18.667 \\
C. melanadenia Zapotitlán & 5 & 9 & 8 & 5 & 1.0 & 0.001 & 3.8 \\
C. epifanioi Mezcala & 6 & 12 & 7 & 6 & 1.0 & 0.002 & 5.067 \\
C. pannosa & 12 & 32 & 11 & 12 & 1.0 & 0.004 & 9.591 \\
\hline
\end{tabular}

variaron de 0.006 en Huajuapan (C. oyamae) a 0.010 en Playa Azul (C. hintonii) y Zirándaro (C. macvaughii). Caesalpinia oyamae posee mayores valores de diversidad haplotídica $(\mathrm{Hd}=0.980)$ y nucleotídica $(\pi=0.006)$ que $C$. hintonii $(\mathrm{Hd}=0.787, \pi=0.002)$.

El número promedio de diferencias nucleotídicas $(\mathrm{K})$ entre las poblaciones de C. oyamae oscilaron de 2.667 en Tlayahualco a 10.667 en Huajuapan, y en $C$. hintonii, de 3.750 en Zicuítaro a 9.0 en Playa Azul. Entre las especies, C. macvaughii tiene el valor más alto de diferencias nucleotídicas $(\mathrm{K}=11.524)$ y $C$. melanadenia el más bajo $(\mathrm{K}=3.8)$.

Estructura genética y flujo génico. El flujo génico entre $C$. hintonii y $C$. oyamae es bajo $(\mathrm{Nm}=0.26)$, las poblaciones de ambas especies están genéticamente diferenciadas $\left(\mathrm{F}_{\mathrm{ST}}=0.661\right)$. Aproximadamente el $72 \%$ de la variación molecular en $C$. oyamae puede atribuirse a las diferencias entre poblaciones (valores de $\mathrm{F}_{\mathrm{ST}}$ ) y al bajo flujo génico $(\mathrm{Nm}=0.19)$ entre ellas. Los valores $\mathrm{F}_{\mathrm{ST}}$ para todas las comparaciones entre poblaciones de $C$. oyamae fueron altos, excepto para el par Tlayahualco-Tehuitzingo $\left(\mathrm{F}_{\mathrm{ST}}=0.27\right)$. Las poblaciones de $C$. oyamae mostraron diferenciación $\left(\mathrm{F}_{\mathrm{ST}}=0.638\right)$ y bajos valores de flujo génico $(\mathrm{Nm}=0.28)$, excepto para el par Tlayahualco-Tehuitzingo $(\mathrm{Nm}=1.35)$. Zicuítaro e Infiernillo $(C$. hintonii) poseen un valor alto de flujo génico entre ellas $(\mathrm{Nm}=2.51)$ y no presentan diferenciación en el nivel molecular $\left(\mathrm{F}_{\mathrm{ST}}=0.021\right)$ (Cuadro 2).

Las comparaciones pareadas de AMOVA (Cuadro 3) entre $C$. hintonii y $C$. oyamae indican una estructura genética significativa entre ellas para todos los marcadores de plastidio $\left(\Phi_{\mathrm{ST}}=0.314 \mathrm{trn} L, \Phi_{\mathrm{ST}}=0.363 \mathrm{trn} L-F, \Phi_{\mathrm{ST}}=0.982\right.$ psbA-trnH). No se estimó el $\Phi_{\mathrm{ST}}$ entre Valerio Trujano y las otras poblaciones de $C$. oyamae debido a la separación filogenética que mostraron entre ellas. Todos los valores revelaron un patrón significativo de estructura genética $\left(\Phi_{\mathrm{ST}}=0.577\right.$ accD-psaI a $\Phi_{\mathrm{ST}}=0.936$ psbA-trnH $)$.

Redes de haplotipos. En el estudio de los haplotipos de este complejo se establecen 2 grandes regiones denominadas porción oriental y porción occidental (Toledo, 1982). La porción este (oriental) comprende parte de la cuenca media y alta de la DRB y el VTC, y la porción oeste (occidental), desde la parte occidental de la sierra de Taxco-Teloloapan en la cuenca media hasta la cuenca baja del DRB.

En la porción oeste (occidental) se encontraron 14 haplotipos para los 23 individuos de las 2 especies analizadas (C. hintonii y C. macvaughii); el número de mutaciones en el árbol más corto fue de 59. Los individuos de las poblaciones Infiernillo y Zicuítaro de C. hintonii son los que presentaron el menor número de cambios mutacionales entre ellos, compartiendo haplotipos. Los individuos de las poblaciones Zirándaro y Descansadero 
Cuadro 2. Medidas de flujo génico $\left(\mathrm{F}_{\mathrm{ST},} \mathrm{Nm}\right)$

\begin{tabular}{llll}
\hline \multicolumn{1}{c}{ Población 1 } & Población 2 & $F_{S T}$ & Nm \\
\hline C. oyamae & & 0.638 & 0.28 \\
Valerio Trujano & Tlayahualco & 0.729 & 0.19 \\
Valerio Trujano & Tehuitzingo & 0.691 & 0.22 \\
Valerio Trujano & Huajuapan & 0.726 & 0.16 \\
Tlayahualco & Tehuitzingo & 0.27 & 1.35 \\
Tlayahualco & Huajuapan & 0.531 & 0.17 \\
Tehuitzingo & Huajuapan & 0.477 & 0.18 \\
C. hintonii & & 0.021 & 22.25 \\
Infiernillo & Zicuítaro & 0.021 & 2.51 \\
C. macvaughii & & 0.261 & 1.41 \\
Descansadero & Zirándaro & 0.261 & 1.41 \\
C. hintonii & C. oyamae & 0.661 & 0.26 \\
C. macvaughii & C. hintonii & 0.466 & 0.57 \\
Descansadero & Infiernillo & 0.455 & 0.48 \\
Descansadero & Zicuítaro & 0.520 & 0.42 \\
Zirándaro & Infiernillo & 0.519 & 0.46 \\
Zirándaro & Zicuítaro & 0.636 & 0.28 \\
\hline
\end{tabular}

de C. macvaughii presentaron el mayor número de cambios entre individuos (Fig. 2A).

En la porción Este (oriental) se encontraron 32 haplotipos en las 3 especies de Caesalpinia (C. epifanoi, C. melanadenia y C. oyamae) evaluando 36 individuos; el número de mutaciones en el árbol más corto fue de 134. Los individuos de la población Huajuapan (Oaxaca) de $C$. oyamae son los que presentan el mayor número de cambios mutacionales entre sí, mientras que la población Tlayahualco (Guerrero) es la que muestra el menor número de cambios entre individuos. No se compartieron haplotipos entre las diferentes poblaciones y especies. La red de haplotipos muestra la separación de la población Valerio Trujano (Guerrero) de C. oyamae de las otras poblaciones de su misma especie por 18 pasos mutacionales (Fig. 2 B).

Origen del complejo $C$. hintonii. El análisis de secuencias de las regiones de plastidio dio como resultado árboles con $\mathrm{L}=1107, \mathrm{CI}=0.78$ y $\mathrm{RI}=0.74$ (Fig. 3). El modelo que más se ajustó a los datos obtenidos fue TVM+I+G (frecuencia de las bases variable, transversiones variables y transiciones iguales con distribución gamma y sitios invariables), con los siguientes parámetros: LsetBase=

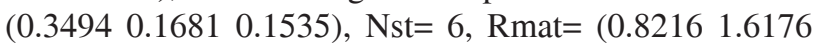
$0.27510 .74931 .6176)$, Rates $=$ gamma shape $=0.6267$, Pinvar $=0.2423$. La estimación de las frecuencias de las bases fueron A: 0.339290; C: 0.172620; G: 0.171272; T: 0.316818. El - Lnlikelihood=0.3150.33587 y la proporción de sitios invariables fue de 0.79 y su valor de desviación estándard es (SDV 5.2725).

La edad estimada del ancestro del complejo C. hintonii y C. pannosa fue de 27.83 Ma (Cnaudroniano, Oligoceno), la edad del complejo se estimó en $22.11 \mathrm{Ma}$ (Aquitaniano, Arikareano, Mioceno), mientras que la divergencia del ancestro de C. pannosa se estimó en $24.19 \mathrm{Ma}$ (OrellanoWnitneyano, Oligoceno) (Fig. 3). Finalmente, se separaron los linajes del complejo $C$. hintonii en las porciones Este y Oeste del río Balsas hace $20.35 \mathrm{Ma}$ (Burdigaliano, Mioceno) y 19.84 Ma (Burdigaliano, Mioceno), respectivamente.

En la porción occidental de la depresión, las edades fueron 16.56 Ma (Burdigaliano, Mioceno) para C. epifanioi, 14.8 Ma (Langhiano, Mioceno) para C. oyamae y $6.98 \mathrm{Ma}$ (Messiniano, Mioceno) para C. melanadenia del VTC. En la porción oriental del Balsas la edad para $C$. hintonii fue de $6.83 \mathrm{Ma}$ (Messiano, Mioceno) y para los 2 linajes de $C$. macvaughii las edades fueron $8.36 \mathrm{Ma}$ (Zirándaro de los Chávez, Guerrero) y $8.65 \mathrm{Ma}$ (Descansadero, Nueva Italia, San Francisco y la Huacana, Michoacán), ambas edades pertenecen al Tortoniano, Mioceno.

\section{Discusión}

Asociación de la estructura geográfica y los factores históricos. La red de haplotipos claramente muestra que las poblaciones de $C$. oyamae están separadas en 3 linajes que no sobreponen sus distribuciones geográficas. La estructura geográfica está bien apoyada por el $72 \%$ del total de la variación en las 4 regiones de plastidio (análisis de AMOVA) y es claro que existe escasa variabilidad entre poblaciones dentro de cada uno de esos linajes (6\%). El AMOVA y la diferenciación genética indican que existe diferenciación significativa entre las distintas poblaciones de $C$. oyamae. El flujo génico entre las poblaciones de Valerio Trujano y Huajuapan es restringido, encontrándose 2 linajes divergentes. Los niveles de flujo génico inferidos pueden ser el resultado de la interacción entre diversos factores bióticos, así como selección diferencial de los polinizadores, debido a que el tamaño y densidad poblacional varían, así como el color y tamaño de las flores (Sotuyo et al., 2004).

En el caso de C. pannosa, la especie es polimórfica variando en tamaño, indumento y densidad de glándulas; bajo este taxon fueron sinonimizadas $C$. arenosa, $C$. californica y C. mexicana var. californica que se distribuyen desde San José del Cabo hasta Comondú (Lewis, 1998). La región de El Cabo y el resto de la península tienen orígenes distintos y hubo una expansión de los desiertos de la parte media de la península hacia las zonas del sur 
Cuadro 3. Análisis de varianza molecular (estadísticos $\Phi$ ) en el complejo $C$. hintonii. Las distancias genéticas entre los haplotipos se calcularon de acuerdo con los estadísticos F a partir de las frecuencias haplotípicas. Para evaluar si los índices de fijación eran significativos se realizaron 10000 permutaciones no paramétricas en los grupos, poblaciones y haplotipos $* * * \mathrm{P}<0.0001$. $C E P=C$. epifanioi, $C H=C$. hintonii, $C M C=C$. macvaughii, $C M E=C$. melanadenia y $C O=C$. oyamae

\begin{tabular}{|c|c|c|c|c|c|c|c|c|c|}
\hline $\begin{array}{l}\text { Región } \\
\text { trnL }\end{array}$ & $\begin{array}{c}C H v s \\
C O\end{array}$ & $\begin{array}{c}C H \text { vs } \\
C M C\end{array}$ & $\begin{array}{c}C H v s \\
C E\end{array}$ & $\begin{array}{l}C H \text { vs } \\
C M E\end{array}$ & $\begin{array}{l}C H v s \\
C M C\end{array}$ & $\begin{array}{l}C O v s \\
C M E\end{array}$ & $\begin{array}{c}C O v s \\
C E P\end{array}$ & $\begin{array}{c}C M C v s \\
C M E\end{array}$ & $\begin{array}{l}C M C v s \\
C E\end{array}$ \\
\hline$\Phi_{\mathrm{CT}}$ & 0.150 & 0.077 & -0.196 & -0.026 & 0.498 & 0.030 & $-0.181 * * *$ & 0.600 & 0.509 \\
\hline$\Phi_{\mathrm{SC}}$ & 0.193 & $0.718 * * *$ & $0.725 * * *$ & $0.747 * * *$ & $0.464 * * *$ & $0.550 * * *$ & $0.516 * * *$ & 0.697 & 0.495 \\
\hline$\Phi_{\mathrm{ST}}$ & $0.314 * * *$ & $0.740 * * *$ & $0.671 * * *$ & $0.741 * * *$ & $0.731 * * *$ & $0.564 * * *$ & $0.428 * * *$ & $0.879 * * *$ & $0.752 * * *$ \\
\hline \multicolumn{10}{|l|}{$\operatorname{trn} L-F$} \\
\hline$\Phi_{\mathrm{CT}}$ & 0.027 & 0.298 & -0.158 & -0.300 & 0.233 & 0.169 & 0.370 & 0.934 & 0.940 \\
\hline$\Phi_{\mathrm{SC}}$ & $0.345 * * *$ & $0.908 * * *$ & $0.919 * * *$ & $0.917 * * *$ & 0.366 & $0.796 * * *$ & $0.805 * * *$ & 0.136 & 0.173 \\
\hline$\Phi_{\mathrm{ST}}$ & $0.363 * * *$ & $0.935 * * *$ & $0.907 * * *$ & $0.892 * * *$ & 0.514 & $0.831 * * *$ & $0.877 * * *$ & $0.943 * * *$ & $0.950 * * *$ \\
\hline \multicolumn{10}{|c|}{ accD-psaI } \\
\hline$\Phi_{\mathrm{CT}}$ & $0 * * *$ & 0.655 & 0.036 & -0.264 & 0.490 & -0.193 & 0.223 & 0.034 & 0.169 \\
\hline$\Phi_{\mathrm{SC}}$ & $0 * * *$ & $0.655 * * *$ & $0.712 * * *$ & $0.687 * * *$ & 0.277 & $0.524 * * *$ & 0.565 & 0.326 & 0.443 \\
\hline$\Phi_{\mathrm{ST}}$ & $0 * * *$ & $0.656 * * *$ & $0.723 * * *$ & $0.605 * * *$ & $0.631 * * *$ & $0.432 * * *$ & 0.662 & 0.349 & $0.537 * * *$ \\
\hline \multicolumn{10}{|c|}{ psbA-trnH } \\
\hline$\Phi_{\mathrm{CT}}$ & 0.720 & 0.188 & -0.240 & -0.260 & 0.279 & -0.820 & -0.861 & -0.240 & 0.949 \\
\hline$\Phi_{\mathrm{SC}}$ & $0.935 * * *$ & $0.958 * * *$ & $0.972 * * *$ & $0.975 * * *$ & 0.329 & $0.960 * * *$ & $0.953 * * *$ & $0.972 * * *$ & 0.095 \\
\hline$\Phi_{\mathrm{ST}}$ & $0.982 * * *$ & $0.966 * * *$ & $0.966^{* * *} *$ & $0.969 * * *$ & 0.516 & $0.928 * * *$ & $0.912 * * *$ & $0.966 * * *$ & $0.954 * * *$ \\
\hline \multicolumn{10}{|l|}{ Global } \\
\hline$\Phi_{\mathrm{CT}}$ & 0.00 & -0.00 & -0.00 & -0.00 & -0.00 & 0.0 & -0.00 & -0.00 & -0.00 \\
\hline$\Phi_{\mathrm{SC}}$ & -0.200 & -0.200 & -0.200 & -0.200 & -0.200 & -0.200 & -0.200 & -0.200 & -0.200 \\
\hline$\Phi_{\mathrm{ST}}$ & -0.200 & -0.200 & -0.200 & -0.200 & -0.200 & -0.200 & -0.200 & -0.200 & -0.200 \\
\hline
\end{tabular}

(Grismer, 1994) lo que seguramente ha repercutido en el polimorfismo encontrado en la especie.

Nuestros resultados coinciden con los de Riddle et al. (2000) en la península de Baja California, donde encontraron taxa crípticos geográficamente separados. Esta coincidencia sugiere que los cambios climáticos dejaron similitudes en los patrones de fragmentación y rutas de migración en las especies de zonas desérticas así como en las de bosques secos de la península. Los estudios filogeográficos de vertebrados de los desiertos mexicanos muestran eventos históricos repetidos de divergencia consistentes con los eventos vicariantes propuestos desde el Mioceno hasta el Pleistoceno temprano (Schmidtly et al., 1993; Riddle y Hafner, 2006). Caesalpinia pannosa es parte de una de esas interrupciones filogeográficas, al ser el taxon hermano del complejo $C$. hintonii. Parecería que un evento mayor en el pasado ocasionó la divergencia genética en la fauna y flora de Baja California. Debido a la divergencia que se ha encontrado en otros taxa (e.g., Riddle et al., 2000; Crews y Hedin, 2006) es probable que un solo evento vicariante, o bien, una serie de eventos estrechamente relacionados hayan ocurrido en la misma área.

La separación morfológica de las distintas especies es clara, aunque la diferenciación de $C$. oyamae en distintos linajes no lo es, pues no existen diferencias 


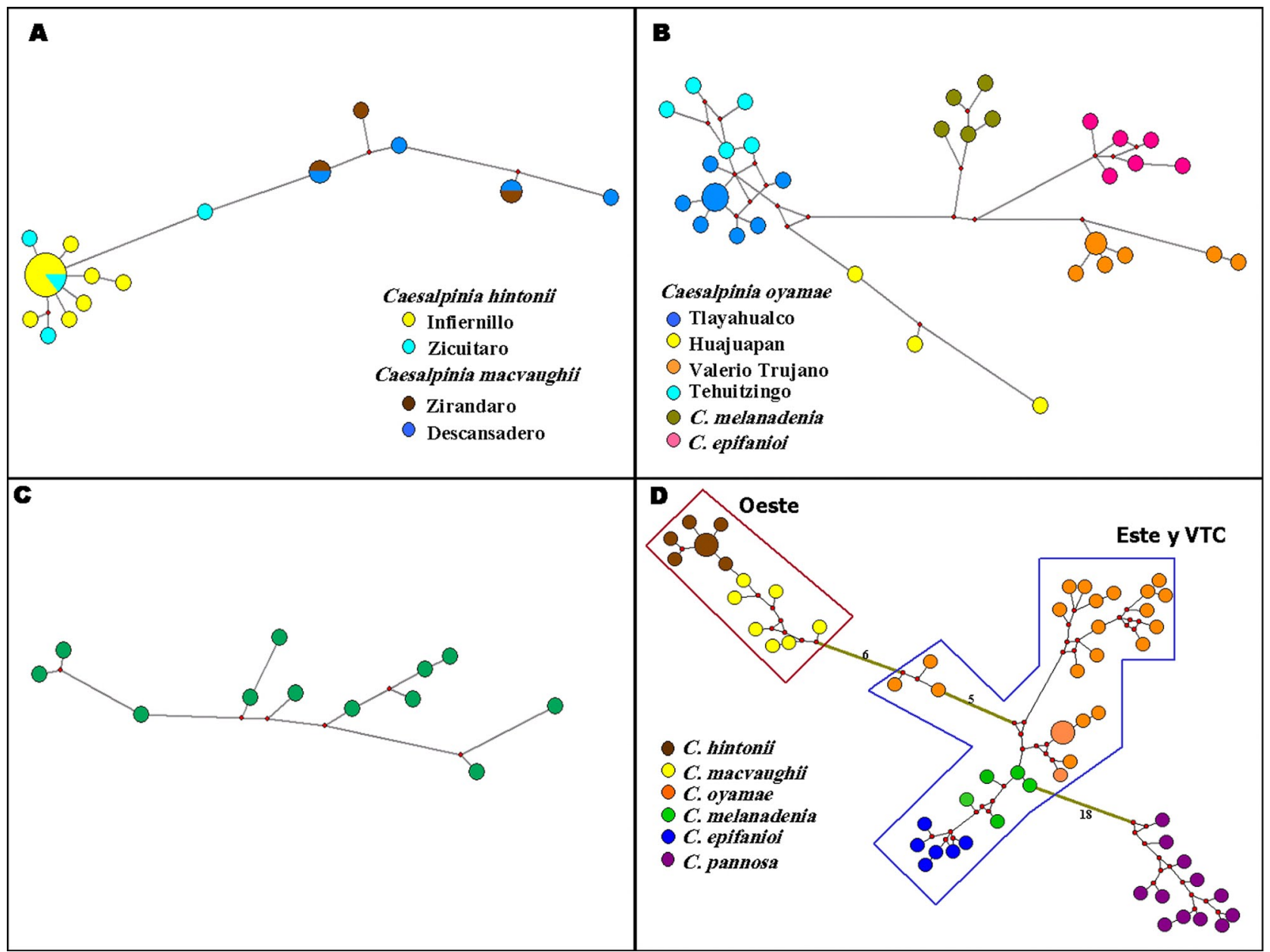

Figura 2. Redes de haplotipos generadas para las especies del complejo Caesalpinia hintonii y el grupo hermano C. pannosa. A, porción oeste de la depresión del río Balsas; B, porción este de la DRB; C. C. pannosa, península de Baja California; D, red de haplotipos global. Los puntos rojos corresponden a haplotipos no muestreados. El tamaño de los círculos es proporcional a la frecuencia de haplotipos.

morfológicas evidentes para separarlos. Sin embargo, se podrían dar 2 explicaciones para los patrones de divergencia observados. La primera hipótesis implicaría una barrera permanente entre las poblaciones de Valerio Trujano y las demás (Tehuitzingo, Tlayahualco y Huajuapan); de tal modo que la vicarianza podría haber generado especiación. Bajo esta misma hipótesis, el patrón observado en $C$. oyamae es consistente con una reciente divergencia, ya que las diferencias morfológicas y moleculares aún no se han acentuado, lo que se esperaría para taxa separados de tiempo atrás. La segunda hipótesis implica una barrera no permanente, aquí las diferencias en los patrones de divergencia deberían estar reflejando diferencias en habilidades de dispersión o de polen. Patrones de divergencia similares en taxa distribuidos en las mismas localidades podrían ayudar a discernir entre estas 2 alternativas. Una evidencia a favor de la barrera permanente es que no se puede reconocer divergencia morfológica en las especies de esta región, mientras que las regiones de plastidio revelan una clara divergencia entre esos haplotipos.

Aislamiento periférico. De acuerdo con el modelo de especiación peripátrica, la deriva génica pudo haberse acentuado en la periferia de la distribución de la especie debido al pequeño tamaño poblacional, el flujo génico restringido y la adaptación a condiciones subóptimas (Carson y Templeton, 1984). Dichas diferencias en el arreglo espacial y el número de individuos en la población de la Caesalpinia ancestral pudieron haber desencadenado la divergencia de $C$. epifanoi y $C$. melanadenia y, la deriva génica pudo ser la responsable de acentuar las diferencias genéticas y morfológicas en estas especies. Los resultados 


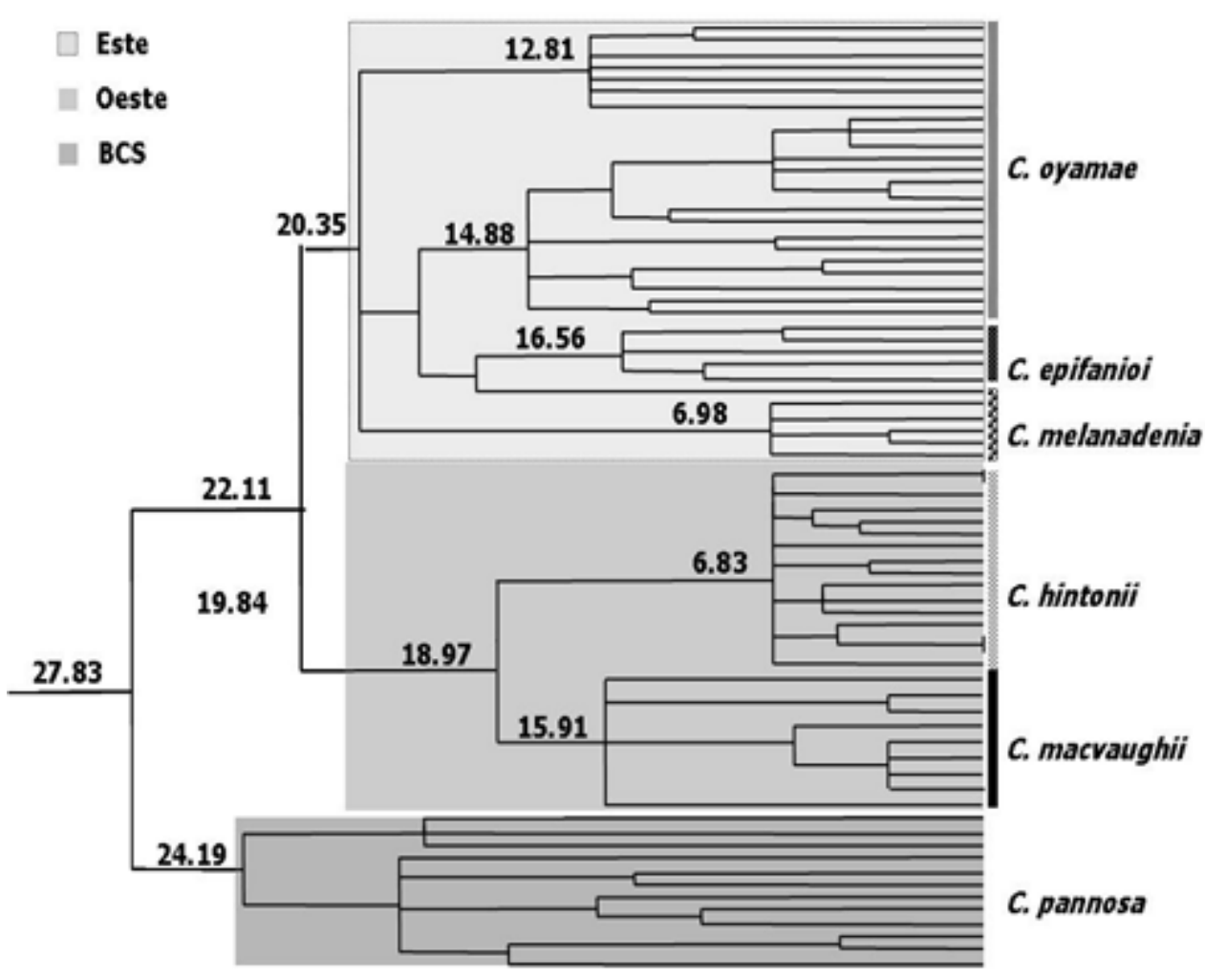

Figura 3. Árbol NPRS (nonparametric rate smoothing) del complejo Caesalpinia hintonii y C. pannosa, donde se muestran las edades aproximadas de divergencia de las distintas especies y poblaciones; así como la separación de las porciones de la DRBVTC. Arriba de las ramas, edades de los grupos.

del AMOVA y el aislamiento geográfico indican que el flujo génico entre estas especies y lo que se conoció como $C$. hintonii, sensu lato (actualmente $C$. hintonii y las diferentes poblaciones de $C$. oyamae), es bajo. De la misma manera, la población Valerio Trujano de $C$. oyamae muestra un patrón de estructura genética y bajos niveles de flujo génico con sus poblaciones conespecíficas, el cual probablemente fue suficiente para desencadenar la diferenciación mediada por deriva génica.

Origen del complejo Caesalpinia hintonii. Contrariamente a la premisa de que el complejo $C$. hintonii tuvo un origen reciente (Sotuyo et al., 2007), el fechamiento preliminar muestra que a partir del Oligoceno tardío y hasta poco antes del Plioceno las poblaciones y especies del complejo se diversificaron.

En la península de Baja California las poblaciones de la endémica $C$. pannosa divergieron, originando el polimorfismo en tamaño, indumento y densidad de glándulas que conocemos ahora (Lewis, 1998). La península de Baja California fue separada de tierra firme hacia finales del Mioceno (10-5 Ma; Oskin et al., 2001). La filogenia fechada para Bursera sugiere que las especies residentes en esas zonas estaban separadas de sus congéneres antes de la separación de la península de Baja California (Becerra, 2005). Lo mismo encontramos en $C$. pannosa (24.19), que fue separada antes de dicho evento vicariante. Por otro lado, para explicar la formación del complejso $C$. hintonii se debe incluir un evento vicariante durante el cual se separan $C$. pannosa y el ancestro del complejo C. hintonii hace aproximadamente $27.83 \mathrm{Ma}$. El evento puede relacionarse con la construcción de la sierra Madre Occidental, cadena volcánica producto de un intenso vulcanismo explosivo (el más grande del Cenozoico) que creó una meseta de rocas ignimbríticas con elevaciones superiores a los $2000 \mathrm{~m}$, durante un lapso de pocos millones de años (Ferrari et al., 2005). El emplazamiento de estas rocas creó una barrera topográfica en dirección NNO-SSE desde la frontera con los Estados Unidos de América, en Sonora hasta Guadalajara, Jalisco. Este vulcanismo se prolongó al sur de la FTVM hasta la región comprendida entre la Presa el Infiernillo y Taxco (Ferrari et al., 2002; Ferrari et al., 2005). La construcción de la sierra Madre Occidental debió haber afectado la circulación atmosférica provocando la creación de un clima distinto entre Baja California y el resto de México (Gómez-Tuena et al., 2005) La fase principal del vulcanismo de la sierra Madre Occidental se terminó aproximadamente a los 28 Ma (Ferrari et al., 2005), edad que coincide con el evento vicariante de separación entre C. pannosa y el ancestro del complejo de C. hintonii. 
Por su parte, el complejo $C$. hintonii inició su divergencia, en la ya constituida depresión del río Balsas, hace 22.11 Ma. La depresión posee 2 tipos de geomorfología y de clima, pero no existe un evento geológico evidente para la separación de ambos linajes en la zona comprendida entre los $99^{\circ} 36^{\prime}$ y los $100^{\circ} 51^{\prime}$. El vulcanismo en esta región de separación terminó antes, aproximadamente $30 \mathrm{Ma}$ (Cerca-Martínez et al., 2007), y la deformación orogénica que provocó la formación de las montañas NS (Sierra de Taxco-Teloloapan) en la zona data de hace 80-70 Ma (Cabral-Cano et al., 2000; CercaMartínez et al., 2007). Sin embargo, una posibilidad es que el levantamiento de una cadena montañosa que bordea la cuenca del Balsas, al sur de esta región, haya influido sobre este proceso divergente en el complejo. Dicho levantamiento es de suma importancia, ya que en la zona se encuentran expuestas rocas que se formaron entre los 15 y $20 \mathrm{~km}$ de profundidad (Morán-Zenteno et al., 1996). Aunque la edad en que fueron exhumadas estas rocas no se conoce con precisión, las reconstrucciones regionales indican que debió haber ocurrido antes del principio del Mioceno (Aquitaniano, 23.7-20 Ma; Ducea et al., 2004) lo que coincide con la edad estimada de divergencia en el complejo.

Existen, a la vez, varios eventos del Mioceno medio (16-11 Ma) que coinciden con el establecimiento de la FVTM al norte de la región. Durante el Mioceno temprano, el arco volcánico de la sierra Madre Occidental rota aproximadamente $30^{\circ}$ en sentido de las manecillas del reloj, acercándose a Baja California (Ferrari et al., 1999) y el vulcanismo se extiende hasta el noroeste de Oaxaca (Ferrusquía-Villafranca, 1993); durante el Mioceno tardío (11.2-5.3), el arco de la FVTM alcanza una orientación estable. Esta barrera de dirección EO pudo causar un cambio de clima y probablemente generó microclimas más al sur influyendo en la divergencia poblacional de las distintas especies del complejo en las porciones oriental y occidental del Balsas.

De acuerdo con Becerra (2005), el género Bursera tuvo su mayor tasa de especiación hace 26-20 Ma, lo cual coincide con el último pulso volcánico de la sierra Madre Occidental (Ferrari et al., 2002). Dichos datos coinciden también con lo que encontramos en el complejo de $C$. hintonii, donde el origen y diversificación se da entre los 27.83 y 20.35 Ma. Becerra (2005) encuentra también que después de los 13.5 Ma decreció la diversificación en Bursera, lamentablemente no existen datos de genética de poblaciones en el género como para compararlos con los encontrados en el complejo $C$. hintonii.

Consideraciones finales. El complejo C. hintonii es el primer grupo de especies analizadas filogeográficamente en la región de la DRB y en el VTC. Los niveles de divergencia en las 4 regiones de plastidio analizadas son mucho mayores a las que típicamente se encuentran dentro de las poblaciones de una misma especie.

En C. oyamae se formaron haplogrupos distintos, donde la carencia de haplotipos compartidos podría ser evidencia del aislamiento reproductivo, dado sus bajos niveles de flujo génico (Cuadro 2). A pesar de la variación molecular, las poblaciones de C. oyamae exhiben escasa o nula variación fenotípica entre ellas. Probablemente, su similitud refleja no sólo una ancestría común reciente sino la acción de selección estabilizadora para producir este ensamble mimético. Al contrario de lo que se esperaba debido a la similitud morfológica, las poblaciones de $C$. oyamae y $C$. macvaughii muestran mayor divergencia en las secuencias que otros pares de especies congenéricas. Lo opuesto sucede con $C$. hintonii, para la que se describieron 2 variantes morfológicas (Contreras, 1991) y donde la divergencia molecular es menor. Los haplogrupos encontrados en C. oyamae y C. macvaughii no pueden describirse formalmente como especies, ya que a pesar de que el empleo de las 4 regiones de plastidio facilitó el reconocimiento de estos linajes, los caracteres morfológicos que hasta ahora hemos evaluado no muestran diferencia alguna entre ellos (Sotuyo, 2007) y, de no ser que incluyamos la distribución geográfica, no existe manera alguna de distinguirlas. No sólo eso, es indudable, que una delimitación de estas entidades requiere de un análisis de marcadores nucleares que permitiría un mayor conocimiento de estas especies. Sin embargo, a pesar de que los linajes encontrados en C. oyamae y C. macvaughii no adquieran la categoría de especie, la genealogía de éstas y de otras especies pueden proporcionar información invaluable acerca de si el vulcanismo asociado a la FVTM dio origen a la gran cantidad de especies en la DRB y en el VTC. La genealogía del complejo $C$. hintonii sugiere una ruptura filogeográfica entre los linajes maternos dentro de las 2 porciones (Este y Oeste) de la depresión del río Balsas.

Las edades estimadas para el complejo coinciden con otros estudios filogeográficos sobre taxa de vertebrados en los desiertos cálidos de México que han mostrado eventos históricos de divergencia repetidos, consistentes con los eventos vicariantes propuestos para el MiocenoPleistoceno temprano (Schmidly et al., 1993; Riddle y Hafner, 2006). Lo que aún queda por contestar es qué está o qué ha pasado durante los últimos 10 millones de años, donde aparentemente no hay divergencia en dichas especies, mientras que grupos como Phaseolus diversificaron en más de 60 especies, en aproximadamente el mismo lapso (Delgado Salinas et al., 2006). 


\section{Agradecimientos}

El primer autor agradece a Lola Lledó y a Félix Forest (RBG, Kew) por su ayuda en las calibraciones. El presente trabajo fue posible gracias a los apoyos otorgados a Solange Sotuyo por parte de CONACYT (beca 15291811144), DGEP-UNAM y Royal Botanic Gardens, Kew. Agradecemos los comentarios del editor Daniel Piñero y de los revisores anónimos que enriquecieron el manuscrito .

\section{Literatura citada}

Bandelt, H. J., P. Forster y A. Röhl. 1999. Median-joining networks for inferring intraspecific phylogenies. Molecular Biology and Evolution 16:37-48.

Becerra, X. J. y L. Venable. 1999. Nuclear ribosomal DNA and its implications for evolutionary trends in Mexican Bursera (Burseraceae). American Journal of Botany. 86:1047-1057.

Becerra, X. J. 2005. Timing the origin and expansion of the Mexican tropical dry forest. Proceedings of the National Academy of Sciences 102:19019-10932.

Beristain, O., A. Valiente-Banuet, A. Dávila y R. Medina. 1996. Tipos de vegetación y diversidad $\beta$ en el valle de Zapotitlán de las Salinas, Puebla, México. Boletín de la Sociedad Botánica de México 59:35-58.

Cabral-Cano E., H. R. Lang, C. G. y A. Harrison. 2000. Stratigraphic assessment of the Arcelia-Teloloapan area, southern Mexico: implications for southern Mexico's postNeocomian tectonic evolution. American Earth Science 13:443-457.

Contreras, J. L. 1991. Contribución al conocimiento del género Caesalpinia (Leguminosae: Caesalpinioideae) en el estado de Guerrero. Tesis, Facultad de Ciencias, Universidad Nacional Autónoma de México, México, D. F. 136 p.

Carson, H. L. y A. R. Templeton. 1984. Genetic revolutions in relation to speciation phenomenon: the founding of new populations. Annual Review of Ecology and Systematics 15:97-131.

Cerca-Martínez, M., L. Ferrari, M. López-Martínez, B. Martiny y A. Iriondo. 2007. Late Cretaceous shortening and early Tertiary shearing in the central Sierra Madre del Sur, southern Mexico: insights into the evolution of the Caribbean-North America plate interaction. Tectonics 26: TC3007, 10.1029/2006TC001981.

Crews, S. C. y M. Hedin. 2006. Studies of morphological and molecular phylogenetic divergence in spiders (Araneae: Homalonychus) from the American southwest, including divergence along the Baja California Peninsula. Molecular Phylogenetics and Evolution 38:470-478.

Delgado-Salinas, A., R. Bibler y M. Lavin. 2006. Phylogeny of the genus Phaseolus (Leguminosae): a recent diversification in an ancient landscape. Systematic Botany 31:779-791.

Ducea, M. N., V. A. Valencia, S. Shoemaker, P. Reiners, P. DeCelles, M. Campa, D. Moran-Zenteno y J. Ruiz. 2004. Rates of sediment recycling beneath the Acapulco Trench:
Constraints from (UTh)/ He Thermochronology. Journal of Geophysical Research 10.1029/2003JB003112.

Excoffier, L., P. E. Smouse y J. M. Quattro. 1992. Analysis of molecular variance inferred from metric distances among DNA haplotypes - application to human mitochondrial DNA restriction data. Genetics 131:479-491.

Excoffier, L., G. Laval y S. Schneider. 2005. Arlequin ver. 3.0: An integrated software package for population genetics data analysis. Evolutionary Bioinformatics Online 1:47-50.

Felsenstein, J. 1981. Evolutionary trees from gene frequencies and quantitative characters: finding maximum likelihood estimates. Evolution 35:1229-1242.

Ferrari, L., M. López-Martínez, G. Aguirre-Díaz y G. CarrascoNúñez. 1999. Spacetime patterns of Cenozoic arc volcanism in central Mexico: from the sierra Madre Occidental to the Mexican Volcanic Belt. Geology 27:303-306.

Ferrari, L., M. Valencia-Moreno y S. Bryan. 2005. Magmatismo y tectónica en la sierra Madre Occidental y su relación con la evolución de la margen occidental de Norteamérica. Boletín de la Sociedad Geológica Mexicana 57:343-378.

Ferrari, L., M. López-Martínez y J. Rosas-Elguera. 2002. Ignimbrite flare up and deformation in the southern sierra Madre Occidental, western Mexico: implications for the late subduction history of the Farallon plate. Tectonics 21:1-24.

Ferrusquía-Villafranca, I. 1993. Geology of Mexico: a synopsis. In Biological diversity in Mexico. Origins and distribution, A. P. Ramamoorthy, R. Bye, A. Lot y J. Fa (eds.). Oxford University Press, New York. p. 3108.

Gómez-Tuena, A., M. T. Orozco-Esquivel y L. Ferrari. 2005. Petrogénesis ígnea de la Faja Volcánica Trans-Mexicana. Boletín de la Sociedad Geológica Mexicana 57:289-321.

González-Rodríguez, A., J. F. Bain, J. L. Goleen y K. Oyama. 2004. Chloroplast DNA variation in the Quercus affinisQ. laurina complex in Mexico: geographical structure and associations with nuclear and morphological variation. Molecular Ecology 13:3467-3476.

Grismer, L. L. 1994. The origin and evolution of the peninsular herpetofauna of Baja California, Mexico. Herpetological Natural History 2:51-106.

Hamilton, M. B. 1999. Four primer pairs for the amplification of chloroplast intergenic regions with intraspecific variation. Molecular Ecology 8:521-523.

Herendeen, P. S. y D. L. Dilcher. 1991. Caesalpinia subgenus Mezoneuron (Leguminosae: Caesalpinioideae) from Tertiary of North America. American Journal of Botany 78:1-12.

Herendeen, P. S. 1992. The fossil history of the Leguminosae from the Eocene of southeastern North America. In Advances in legume systematics: Part 4. The fossil record, P. S. Herendeen y D. L. Dilcher (eds.). Royal Botanic Gardens, Kew. p. 85-160.

Herendeen, P. S. y P. R. Crane. 1992. Early caesalpinioid fruits from the Palaeogene of southern England. In Advances in legume systematics IV: Fossil Leguminosae, P. S. Herendeen y D. L. Dilcher (eds.) Royal Botanic Gardens, Kew, Richmond, Surrey. p. 57-68.

Hudson, R. R., M. Slatkin y W. P. Maddison. 1992. Estimation of levels of gene flow from DNA sequence data. Genetics 132:583-589. 
Jiménez, R. J. 1979. Estudio florístico de la vegetación en una localidad en la cuenca baja del río Balsas I. Informe mimeografiado. Archivo de la Comisión de Biologías de Campo. Facultad de Ciencias, Universidad Nacional Autónoma de México, México, D.F.

Lavin, M. 2006. Floristic and geographical stability of discontinuous seasonally dry tropical forests explains patterns of plants phylogeny and endemism. In Neotropical savannas and seasonally dry forests, R. T. Pennington, G. P. Lewin y J. A. Ratter (eds.). Plant diversity, biogeography, and conservation, The Systematics Association Special Volume Series 69. Taylor and Francis, Abingdon, Oxford. p. 433-447.

Lewis, G. P. 1998. A revision of the Poincianella Erythrostemon group. Royal Botanic Gardens, Kew, Richmond Surrey. $233 \mathrm{p}$.

Matos, J. A., y B. A. Schaal. 2000. Chloroplast evolution in the Pinus montezumae complex: a coalescent approach to hybridization. Evolution 54: 1218-1233.

Mendenhall, M. 1994. Phylogeny of Baptisia and Thermopsis (Leguminosae) as inferred from chloroplast DNA and nuclear ribosomal DNA sequences, secondary chemistry, and morphology. Ph.D. thesis, University of Texas, Austin.

Morán-Zenteno, D. J., P. Corona-Chávez y G. Tolson. 1996. Uplift and subduction erosion in southwestern Mexico since the Oligocene: Pluton geobarometry constraints. Earth and Planetary Science Letters 141:51-65.

Nason, J. D., J. L. Hamrick y T. H. Fleming. 2002. Historical vicariance and post-glacial colonization effects on the evolution of genetic structure in Lophocereus, a Sonoran desert cactus. Evolution 56:2214-2226.

Nei, M. 1987. Molecular evolutionary genetics. Masatoshi Nei, Columbia, New York. 512. p.

Oskin, M., J. Stock y A. Martín-Barajas. 2001. Rapid localization of Pacific-North American plate motion in the Gulf of California. Geology 29:459-462.

Pennington, R. T., M., Lavin, D. E. Prado, C. A. Pendry, S. Pell, S. y C. A. Butterworth. 2004. Historical climate change and speciation: Neotropical seasonally dry forest plants show patterns of both Tertiary and Quaternary diversification. Philosophical Transactions of the Royal Society 359:515538.

Polzin, T. y S. V. Daneschmand. 2003. On Steiner trees and minimum spanning trees in hypergraphs. Operations Research Letters 31:1220.

Rambaut, A. y M. Charleston. 2000. TreeEdit, ver. 1.0 alpha 10 (http://evolve.zoo.ox.ac.uk/software/TreeEdit/TreeEdit); último acceso: 06.V.2009.

Riddle, B. R., D. J. Hafner, L. F. Alexander y J. R. Jaeger. 2000. Cryptic vicariance in the historical assembly of a Baja California peninsular desert biota. Proceedings of the National Academy of Sciences 97:9740-9745.
Riddle, B. R. y D. J. Hafner. 2006. Biogeografía histórica de los desiertos cálidos de Norteamérica. In Genética y mamíferos mexicanos: presente y futuro, E. Vázquez-Domínguez, D. J. Hafner (eds.). New Mexico Museum of Natural History and Science Bulletin. 32:57-65.

Rozas, J., J. C. Sánchez-Del Barrio, X. Messeguer y R. Rozas. 2003. DnaSP, DNA polymorphism analyses by the coalescent and other methods. Bioinformatics 19:2496-2497.

Sanderson, M. J. 1997. A nonparametric approach to estimating divergence times in the absence of rate constancy. Molecular Biology and Evolution 14:1218-1231.

Schmidtly, D. J., K. T. Wilkins y J. N. Derr. 1993. Biogeography. In Biology of the Heteromyidae, Special publication 10, $\mathrm{H}$. H. Genoways y J. H. Brown (eds.). American Society of Mamalogists, Shippensburg, Pennsylvania. p. 319-356.

Sotuyo, S., J. L. Contreras, A. Delgado-Salinas y K. Oyama. 2004. Genetic structure of the endemic Caesalpinia hintonii complex (Leguminosae: Caesalpinioideae). Plant Systematics and Evolution 247:131-143.

Sotuyo, S. y G. P. Lewis. 2007. A new species of Caesalpinia from the Río Balsas Depression, Mexico, and an updated taxonomic circumscription of the Caesalpinia hintonii complex (Leguminosae: Caesalpinioideae: Caesalpinieae: Poincianella group). Brittonia 59:33-36.

Sotuyo, S., A. Delgado-Salinas, M. W. Chase, G. P. Lewis y K. Oyama. 2007. Cryptic speciation in the Caesalpinia hintonii complex (Leguminosae: Caesalpinioideae) in a seasonally dry Mexican forest. Annals of Botany 100:1307-1314.

Sotuyo, S. 2007. Historia evolutiva del complejo Caesalpinia hintonii (Leguminosae: Caesalpinioideae): filogenia, estructura genética y aislamiento reproductivo. Tesis, doctorado Instituto de Ecología, Universidad Nacional Autónoma de México, México, D.F. 147 p.

Sousa, M. S. y A. Delgado-Salinas. 1993. Mexican Leguminosae: Phytogeography, endemism, and origins. In Biological diversity in Mexico. Origins and distribution Ramamoorthy, A. P., Bye, R., Lot, A., Fa, J. (eds.). Oxford University Press, New York. p. 459-511.

Swofford, D. L. 2002. PAUP*: phylogenetic analysis using parsimony (*and other methods), ver. 4.0b8. Sinauer, Sunderland, Massachusetts.

Taberlet, P., L. Fumagalli, A. G. Wust-Saucy y J. F. Cosson. 1998. Comparative phylogeography and postglacial colonization routes in Europe. Molecular Ecology 7:453-464.

Toledo M., C.A. 1982. El género Bursera (Burseraceae) en el estado de Guerrero (México). Tesis, Facultad de Ciencias, Universidad Nacional Autónoma de México, México, D.F. $200 \mathrm{p}$.

Tovar Sánchez, E. y K. Oyama. 2004. Natural hybridization and hybrid zones between Quercus crassifolia and Quercus crassipes in Mexico. Morphological and molecular evidence. American Journal of Botany 91:1352-1363. 